\title{
Can mechanical circulatory support be an effective treatment for HFpEF patients?
}

\author{
Einar Gude ${ }^{1} \cdot$ Arnt E. Fiane $e^{2,3}$
}

Accepted: 21 July 2021 / Published online: 9 August 2021

(c) The Author(s) 2021

\begin{abstract}
Heart failure with preserved ejection fraction (HFpEF) is increasing in prevalence and represents approximately $50 \%$ of all heart failure (HF) patients. Patients with this complex clinical scenario, characterized by high filling pressures, and reduced cardiac output (CO) associated with progressive multi-organ involvement, have so far not experienced any significant improvement in quality of life or survival with traditional HF treatment. Left ventricular assist devices (LVAD) have offered a new treatment alternative in terminal heart failure patients with reduced ejection fraction (HFrEF), providing a unique combination of significant pressure and volume unloading together with an increase in $\mathrm{CO}$. The small left ventricular cavity in HFpEF patients challenges left-sided pressure unloading, and new anatomical entry points need to be explored for mechanical pressure and volume unloading. Optimized and pressure/volume-adjusted mechanical circulatory support (MCS) devices for HFrEF patients may conceivably be customized for HFpEF anatomy and hemodynamics. We have developed a long-term MCS device for HFpEF patients with atrial unloading in a pulsed algorithm, leading to a significant reduction of filling pressure, maintenance of pulse pressure, and increase in CO demonstrated in animal testing. In this article, we will discuss HFpEF pathology, hemodynamics, and the principles behind our novel MCS device that may improve symptoms and prognosis in HFpEF patients. Data from mock-loop hemolysis studies, acute, and chronic animal studies will be presented.
\end{abstract}

Keywords HFpEF $\cdot$ MCS $\cdot$ Heart failure $\cdot$ Cardiac hemodynamics

\section{Introduction}

Increasing numbers of patients are diagnosed with heart failure (HF), a disease with considerable impact on morbidity and mortality. It is estimated that 26 million patients suffer from $\mathrm{HF}$ and that $1-3 \%$ of adults and $10 \%$ of the population above the age of 70 years have HF. In the USA, approximately 6 million patients live with HF [1,2]. Prevalence is expected to increase by almost $50 \%$ in the next 10 years due to an aging population, but also due to increased comorbidities among younger adults. HF with preserved ejection fraction (HFpEF) has been a focus of concern and is now more frequent among admitted patients than HF with reduced

Einar Gude

egude@ous-hf.no

Dept of Cardiology, Oslo University Hospital, Oslo, Norway

2 Dept of Cardiothoracic Surgery, Oslo University Hospital, Oslo, Norway

3 Faculty of Medicine, University of Oslo, Oslo, Norway ejection fraction (HFrEF) [3, 4]. In HFrEF, left ventricular assist devices (LVADs) have successfully improved symptoms and survival. In this article, we will discuss HFpEF pathology, hemodynamics, and the potential for a novel mechanical circulatory support (MCS) device with unloading from the left atrium (LA) into different outflow sites in the arterial system that may improve symptoms and prognosis in HFpEF patients.

\section{HFpEF, more than a cardiac disease}

The lack of a unified definition and treatment options has made the diagnosis of HFpEF challenging. HFpEF was previously characterized as diastolic dysfunction, typically after non-cardiac causes of dyspnoea were excluded in patients with normal ejection fraction (EF). Today, HFpEF is recognized as a syndrome comprised of multiple cardiometabolic entities with cellular and inflammatory mechanisms leading to diastolic dysfunction, including biventricular systolic impairment, loss of atrial compliance 
associated with atrial arrhythmia, pulmonary vascular disease, loss of adequate chronotropic response, and finally hemodynamic derangement $[5,6]$. Consequently, patients experience pulmonary and venous congestion secondary to high filling pressures and reduced cardiac (CO) output due to low stroke volumes (SV). HFpEF is more than a complex cardiac disease. It includes arterial hypertension and atherosclerotic disease, microvascular inflammation, renal failure (related to hemodynamic impairment, diabetes, and/or medications), and hepatic dysfunction due to congestion or fatty liver. Metabolic abnormalities include visceral adiposity with increased neurohormones and inflammatory cytokines and musculoskeletal degradation (either primary due to HF or secondary to metabolic syndrome and aging) [7]. Females are overrepresented [8]. Some patients experience chest wall restrictions due to loss of mobility. Predominant phenotypes differ across regions in Asia, the USA, and Europe. Whether a single common mechanistic pathway or multiple simultaneous processes lie behind this complex multi-organ pathological scenario is the subject of much discussion, but remains unanswered [9]. In a paper by Escher et al., HFpEF was divided into four phenotypes: (I) genetically inherited hypertrophic cardiomyopathy, (II) infiltrative cardiomyopathy with restrictive physiology, (III) nonhypertrophic cardiomyopathy, (IV) normal ejection fraction with comorbidities (i.e., hypertension, coronary artery disease) and hypertrophy. Dependent of phenotype, deranged hemodynamics caused elevated LA pressure and reduced CO consistent with HFpEF [10]. In this paper, the hemodynamics and physiology, independent of clinical phenotype, will be discussed together with the potential for targeted treatment with MCS in HFpEF.

Mortality in HFpEF is similar to HFrEF patients [11]. The causes of mortality are often more heterogeneous but represent the complexity and consequences of HFpEF and HF with secondary and/or simultaneous affection in other organs [12]. A mortality of $11 \%$ in 2.5 years (NYHA II-III) was demonstrated in the CHARM-preserved trial [13]. Owan et al. demonstrated an equivalent of 2-year mortality of $40 \%$ in both HFpEF and HFrEF among patients admitted with HF symptoms [11]. Amyloidosis, a special subtype of HFpEF caused by deposits of misfolded transthyretin in the extracellular space, has a poor prognosis, with median survival defined by the Gillmore classification between 24 and 69 months depending on levels of brain natriuretic peptide (BNP) above or below $3000 \mathrm{ng} / \mathrm{l}$ and estimated glomerular filtration rate below or above $45 \mathrm{ml} / \mathrm{m} \mathrm{[14]}$. Treatment for cardiac amyloidosis that directly targets the etiology of the disease is available, but unfortunately exclusively for this specific disease among the many etiologies behind HFpEF.

\section{Is HFpEF out of the reach of conventional HF treatment?}

Several trials have tried to target treatment in HFpEF patients and have assessed the etiology of HFpEF (i.e., fibrosis), the hemodynamic derangement (increased filling pressure), or the symptoms (dyspnea, reduced functional capacity) with limited or no success. The European Society of Cardiology (ESC) stated in their HF treatment guidelines from 2016 that no HF medical treatment has demonstrated a reduction in mortality in HFpEF patients $[15,16]$. The primary manifestations of HFpEF are shortness of breath and limited $\mathrm{CO}$ increase during exercise, which represent the treatment paradox in HFpEF. Preload reduction by diuretics improves shortness of breath but possibly reduces $\mathrm{CO}$ and thereby increases fatigue [17]. This is demonstrated in the Aldo-HF study, which demonstrated a reduction in pro-BNP and E/e (filling pressure in the LV/LA by echocardiography), but without effect on functional capacity $\left(\mathrm{VO}_{2}\right)$ [18]. Beta-blockers improve left ventricle (LV) filling time, and also reduce heart rate (HR), which is almost linearly associated with $\mathrm{CO}$, such that reduction may be unfavorable in HFpEF patients due to low SV related to small LV cavity size. On the other hand, reduced HR may improve diastolic filling by increasing the duration of diastole. In practice, the balance between unfavorable reductions in $\mathrm{CO}$ related to reduced $\mathrm{HR}$ and improvements in LV filling varies among individuals.

Beta-blockers are fundamental in HFrEF treatment independent of their effect on HR and have a positive effect on all-cause mortality in patients with $\mathrm{EF}<40 \%$ [19]. HF with EF 40-49\%, which was previously included in HFpEF trials is now defined as heart failure with midrange ejection fraction (HFmrEF) while HFpEF is by ESC guidelines defined as ejection fraction $(\mathrm{EF})>50 \%$ [15]. Four major HFpEF trials (CHARM preserved, I preserved, PET-HF, and TOPCAT) all ended neutral in the primary endpoint $[13,20-22]$. The potential treatment effect of aldosterone in the TOPCAT study and angiotensin/neprilysin inhibitors in the PARAGON study is limited to patients in sub-groups with EF ranging from $40-55 \%$ [23]. These results probably reflect targeting the systolic dysfunction in the HFmrEF more than the true HFpEF pathology. In the PARAGON trial, females and those with lower EF benefitted from angiotensin/neprilysin inhibitor compared to valsartan alone, highlighting the importance of defining normal EF. Normal EF in men is defined as above $50 \%$, but normal EF in women is probably more than $50-55 \%$ [24]. In a study by Wehner et al., mortality demonstrated a $U$-shaped relationship to LVEF with a nadir risk corresponding to LVEF of $60-65 \%$ [25]. This might explain why women had the benefit of medical treatment 
at a higher EF than men and that the treatment effect was preferentially seen in a female population with poorer true systolic function than men, despite equivalent quantified EF [24]. Although these trials may suggest optimism, the data is too scant to define angiotensin-converting enzyme, angiotensin receptor blockers, and angiotensin/neprilysin inhibitors therapy as the salvation for HFpEF patients. The anti-diabetic drug sodium-glucose cotransporter-2 inhibitors, also called gliflozins, are promising in HFpEF and represent a completely new treatment strategy by altering the sodium/glucose channels and inhibit glucose reabsorption in the glomerular filtration.

The optimal treatment for a HFpEF patient would both reduce LA pressure and increase SV, but no such treatment is currently available. Interatrial shunt devices have been tested in HFpEF patients with a reduction of LA pressure and positive short-term outcomes on symptoms. However, these devices do not increase SV [26, 27]. Since many HFpEF patients are older and have a poor quality of life (QoL), endpoints in future HFpEF treatment trials should focus more on well-being in addition to mortality [15]. In HFrEF, LVADs have revolutionized treatment in very sick patients, but LV unloading is not suitable for the HFpEF group due to the small LV cavity size.

\section{How to target HFpEF with mechanical circulatory support?}

LVADs have offered a new treatment alternative in terminal HFrEF patients with the unique combination of significant pressure and volume unloading in combination with an increase in $\mathrm{CO}$, a win-win solution. Optimized and pressure/volume-adjusted solutions for HFrEF patients may conceivably be customized for HFpEF anatomy and pressure/ volume curves.

MCS in HFpEF patients would likely involve alternative and potentially more technically difficult anatomical entry/ inflow sites than the LV, with less robust anatomic structures and lower pressures than in the LV. The higher gradient between inflow and outflow will require more energy to maintain forward flow through the MCS. The side effects of LVADs, including systemic thromboembolism, bleeding, infections, and lack of pulse pressure, would have to be addressed to optimize MCS support in an elderly multimorbid HFpEF population. The placement of a possible MCS device in the HFpEF population must ideally be simplified and surgery minimalized, customized for a fragile patient group. In short, the ideal MCS device for the HFpEF population would be a low-risk, long-term durable, and easily implantable device for a population where destination therapy (DT) is most likely.

\section{Unloading of the LA-consequences for HFpEF hemodynamics}

Burkoff et al. have elegantly demonstrated the hemodynamic effects of unloading LA to the aorta in ex vivo mock-loop simulation tests [28], while the use of The Tandem Heart device (Cardiac Assist Inc., Pittsburg, PA, USA) centrifugal pump in short-term unloading of LA to femoral artery has been proven clinically effective in HF patients by lowering LA pressure with additional systemic circulatory support [29]. For longer term use with the unloading of LA to the right axillary artery, Meyns et al. showed proof of concept with the partial support LVAD CircuLite Synergy Micro-Pump Device, later acquired by HeartWare (Framingham, Mass) and thereafter by Medtronic (Minneapolis, MN, USA) [30]. A novel impeller device, placed in the mitral position and intended to decrease LA pressure and increase LV preload, has been tested in a mock loop as a treatment principle [31]. A non-pulsatile pump in the mitral position will unload the LA in diastole due to pressure gradients between the $\mathrm{LA}$ and the LV; therefore, rotor speed (rpm) must be tuned to avoid retrograde flow through the pump in the systole. Escher et al. elegantly demonstrated the effect of a pneumatic pulsatile pump (CoPulse) in a mock loop with a single cannula in the LV in different HFpEF phenotypes. By CoPulsing with the heartbeat, filling and emptying the pump increased $\mathrm{SV}$ in addition to unloading from LV into the pump in diastole. CoPulse demonstrated the pump's potential to unload the LA and increase $\mathrm{CO}$ differently for the four HFpEF phenotypes and is one of many potential treatment mechanisms relevant for the HFpEF population [10].

Timms et al. investigated the difference in hemodynamics with either LA or LV inflow cannulation in a HFrEF LVAD mock loop. EF, stroke work, and pump flow rates were lower with LA compared to LV cannulation in all HF conditions.

Adequate ventricular ejection remained with atrial cannulation under low levels of mechanical support, however, with risk of thrombus formation in very low EF [32].

Several studies have assessed the hemodynamic perturbations in HFpEF patients at rest and exercise, and understanding invasive properties is essential to unload the LA in HFpEF. LA pressures assessed by pulmonary capillary wedge pressure (PCWP) during right heart catheterization have been measured in healthy and in HFpEF patients [33]. In healthy individuals, LA pressure/PCWP varies from normal pressure at rest $(5-12 \mathrm{mmHg})$ up to $20 \mathrm{mmHg}$ at exhaustion. In a diagnostic exercise algorithm by Berry et al., exercise-induced PCWP $>20 \mathrm{mmHg}$ and a parallel increase in mean pulmonary artery pressure 
(mPAP) (and a more or less unchanged transpulmonary gradient (TRP)) define exercise-induced post-capillary pulmonary hypertension (PH) [34]. Consequently, pulmonary vascular resistance may be normal, depending on the CO response to exercise. Long-standing HFpEF may induce structural changes in the pulmonary vasculature with secondary increased TPG, a complicating factor both in the diagnostic algorithm and the clinical approach to HFpEF treatment. Systolic pressure in the LA depends on atrial and ventricular properties and the degree of mitral regurgitation. V-wave in LA/PCWP can be caused by the direct pressure of LV contraction on the mitral leaflets or by a mitral regurgitation that also induces a volume load to the LA. Reddy et al. demonstrated V-waves in the LA up to $30 \mathrm{mmHg}$ in patients with HFpEF during rest. During supine exercise with simultaneous PCWP measurements, mean pressure was measured up to $30 \mathrm{mmHg}$ with a systolic V-wave up to $50 \mathrm{mmHg}$ at a workload of only $20 \mathrm{~W}$ [35]. Atrial unloading should therefore be possible and clinically meaningful from a hemodynamic point of view.

In 2010, both Meyns and Klotz reported improved hemodynamic conditions with reduced pulmonary pressures and PCWP together with increased $\mathrm{CO}$ during partial unloading with the CircuLite pump (Medtronic) from the LA to the subclavian artery[30,36]. CircuLite is a small non-pulsatile partial circulatory supporting pump with a capacity up to 3 $\mathrm{L} / \mathrm{m}$; consequently, the majority of the unloading presumably occurs during diastole, when the pressure gradient is at its lowest. Neither the effects on flow through the mitral valve and LV filling nor the volume circulating through the CircuLite pump is reported. The aortic flow signals are not reported, and theoretically, an increase in arterial pressure in diastole and a decrease in pressures in systole could be observed. The increase in the cardiac index from $2.0 \pm 0.4$ to $2.7 \pm 0.6 \mathrm{~L} / \mathrm{min}$ demonstrated a limited $\mathrm{CO}$ increase with a corresponding decrease in PCWP from $28.5 \pm 6.0$ to $19.7 \pm 6.9 \mathrm{mmHg}$.

Despite the successful decrease of left side filling pressure and increased $\mathrm{CO}$ reported with the CircuLite, there are several pitfalls which have to be addressed.

1. The LA must be partially unloaded to secure adequate LV filling, avoid LV thrombus, and secure adequate native antegrade aortic and especially coronary and carotid flow. The degree of LV filling and ejection and the need for forwarding aortic flow depends on the outflow graft position. LA unloading must therefore be less the more distant the outflow exit site is positioned. For example, outflow in the proximal ascending aorta as in today's LVAD in HFrEF will secure adequate forward flow in ascending aorta including coronary arteries. Outflow position to the right subclavian artery as in the CircuLite secures antegrade carotid flow, but may cause stasis and turbulent flow in the area were antegrade aortic flow and exit graft flow meet. Outflow positioned in the left subclavian artery may compromise coronary perfusion if overpumping occurs and LV SV is too low. Outflow in the descending aorta requires controlled partial flow with possible fatal consequences in the case of low LV filling and aortic native flow and risk of hypoperfusion of the coronary and cerebral arteries. Favorably, outflow distant to the carotid arteries will reduce cerebral emboli from the pump, one of the major adverse events in traditional LVADs. Inflow and outflow sites determine the length of grafts, a possible modulator of graft thrombosis especially at low flow. Reduced embolus risk in the HeartMate III opens for aspirin-free anticoagulation in the ARIES HMIII trial (NCT 04,069,156). This approach would be even safer with an outflow graft distal to the carotid arteries. LVAD used as a right ventricular assist device with inflow in the right atrium (RA) and outflow to the pulmonary artery induces the possibility of minimal flow through the right ventricle (RV). Despite this dilemma, this approach has been successfully applied with less thrombosis using inflow in RA versus RV [37]. Contrary to systemic circulation, emboli to pulmonary circulation
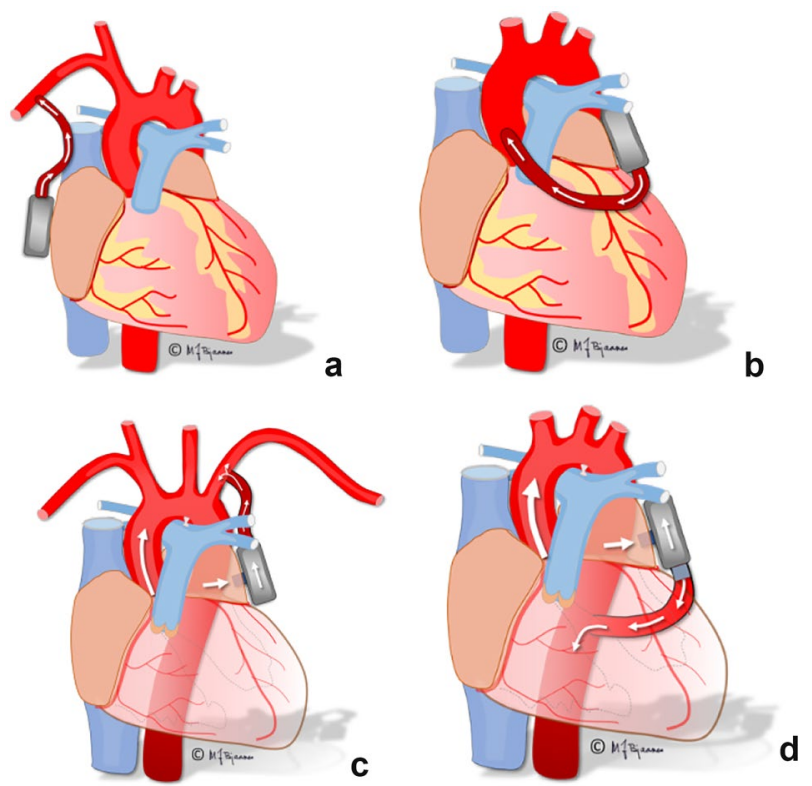

Fig. 1 PulseVAD with pump inflow cannulation of left atrium posterior to groove between right and left atrium and outflow to right subclavian artery a, with pump inflow between left atrial appendage and left pulmonary veins and the outflow graft from the pump connected to ascending aorta $\mathbf{b}$, left subclavian artery $\mathbf{c}$, and descending aorta $\mathbf{d}$. In all sheep studies described, we used strategy d 
have far less severe consequences. Figure 1 demonstrates different outflow sites with inflow from the LA.

2. Unloading of the LA must overcome a higher gradient than traditional LVADs with inflow from the LV. Systolic pressure gradient from the LA (15-40 $\mathrm{mmHg}$ ) to the aorta (100-140/50-100 mmHg) varies, depending on rest or exercise. Most importantly, however, the gradient to overcome is highest in systole, in contrast to traditional LVADs with inflow from LV where the gradient to overcome is highest in diastole. To overcome this gradient, a systolic ramp of pump speed in systole is required to overcome the gradient between inflow and outflow and to unload the greater part of the volume in systole as less unloading has to occur in the diastole. Pulsed MCS may reduce the risk of an embolus from the pump house, mimic natural circulation, and maintain the Windkessel effect which may be positive for end-organ and peripheral perfusion in DT patients [38].

3. LV filling occurs when the mitral valve is open in diastole. LA filling occurs in both systole and diastole, demonstrated by echocardiographic $\mathrm{S}$ and $\mathrm{D}$ flow in the pulmonary veins. LA unloading must preferably occur in systole when the mitral valve is closed. This will also minimize the hemodynamic negative effects of mitral regurgitation. Overpumping may interfere with mitral opening with possible adverse hemodynamic effects. An increase of pump speed in systole to overcome the inflow-outflow gradient and to unload the majority of volume must therefore be planned and timed in a pulsed algorithm.

\section{A new smartpump}

A recently developed pump, PulseVAD (NorthernResearch, Oslo, Norway), is a novel small pulsatile diagonal centrifugal rotary blood pump, using hydrodynamic suspension for magnetically elevated support of a four-blade impeller and partially unloading from the LA to the aorta (Fig. 2). The pump is adaptive to the physiological needs of the patients by use of epicardial ECG and sensor feedback algorithms. Increased pulsed power and increased pump speed in systole are programmed to unload the LA when the mitral valve is closed, maintain pulse pressure, and overcome the increased systolic gradient from the LA to the aorta
Fig. 2 a Physiologic principle of unloading the left atrium to descending aorta. Increase in pump speed during systole to overcome the gradient from left atrium to aorta. b Crosssectional graphic presentation of the PulseVAD. 1. Titanium casing. 2. Hydrodynamically suspended rotor. 3. Inlet tract. 4. Rare earth magnets. 5. Outlet tract. 6. Motor coils

\section{Total flow per cycle $=76 \mathrm{ml}$}

Heat rate 75 beats $/ \mathrm{min} \times 76 \mathrm{ml}=5,61 / \mathrm{min}$
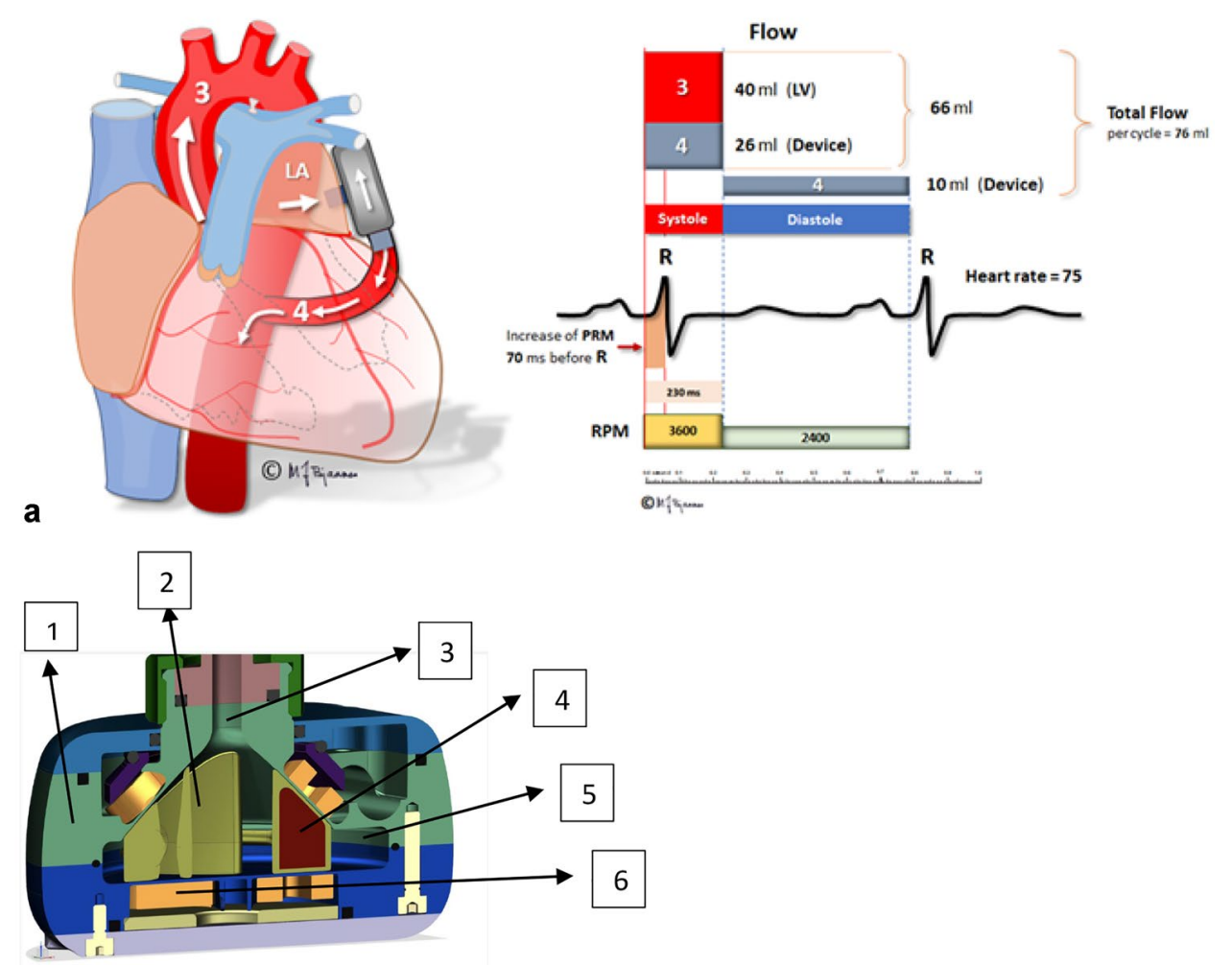
(Fig. 2a). Cross-sectional animation of the pump is presented in Fig. 2b.

Pre-clinical implant testing was performed with human full blood (from six different healthy volunteer medical students) without anticoagulation in a closed ex vivo circuit with PulseVAD. Testing was performed six times during 8-h continuous runs with pulsed pump speed. Hemolysis parameters were analyzed with a simultaneous A and B test before starting the pump and repeated every hour including at test termination. Hemoglobin was targeted to $9-10 \mathrm{~g} / \mathrm{L}$. A gradient of $80 \mathrm{mmHg}$ over the closed circuit was created with a tube clamp distal to the pump outlet. Pressure proximal to the pump was targeted to $10-15 \mathrm{mmHg}$, adjusted by a reservoir. During six tests, 76 time points were analyzed. Plasma-free hemoglobin (PfHb) was $<0.03 \mathrm{~g} / \mathrm{dL}$ in $51 / 81$ $(63 \%)$ of the tests and remaining values were $0.04 \pm 0.05 \mathrm{~g} /$ dL. Lactate dehydrogenase (LDH) was $110 \pm 48 \mathrm{U} / \mathrm{L}$, with no value above $250 \mathrm{U} / \mathrm{L}$. Stable energy consumption less than 4-5 W was observed, with no development of heat. Pump flow was stable between 3 and $5 \mathrm{~L} / \mathrm{min}$. No malfunctions were observed.

Short- and long-term implantations in sheep were performed. Flow probes were attached to the proximal and distal aorta and the outflow graft of the pump. Intravenous lines demonstrated arterial and central venous pressures.

Four short-term acute studies in sedated sheep were performed to test proof of concept. The physiology of the PulseVAD is demonstrated with aortic pressure and flow, pump speed, power, and flow from one of the studies (Fig. 3). Increased rpm during systole demonstrated increased systolic pump flow with a maintained aortic pulse pressure of $90 / 70$ and flow measured with probes and iv lines proximal to the outflow graft. The timing of revolution per minute increase was adjusted to the $\mathrm{R}$ wave of the ECG to optimize the total aortic flow of approximately $5 \mathrm{~L} / \mathrm{min}$ and systolic pressures approximately $100 \mathrm{mmHg}$. Both flow and pressure curves were affected by revolution per minute. Echocardiography was performed simultaneously and ideal optimal pump speed was when full aortic and mitral valve opening were observed. Variable revolution per minute was tested and no suction in the LA was demonstrated with systolic unloading and pump flow up to $4 \mathrm{~L} / \mathrm{min}$ in stable sedated sheep. No supraventricular arrhythmias were observed during induced suction, and rapid loss of suction was demonstrated with a decrease of revolution per minute. Coronary and carotid artery flows were not measured during these tests.

In one long-term study (11 days), revolution per minute of 1900 and unloading of $2-2.5 \mathrm{~L} / \mathrm{m}$ was demonstrated in the theater with sedated sheep. Blood pressure was $75 / 50$ and pulse 90 sinus rhythms. No anticoagulation, anti-platelet drugs, or blood transfusion was administered during the 11-day follow-up. After waking up, the sheep were kept in a crib and monitored with a camera for 11 days without any observed adverse events. Pump parameters were kept unchanged for 11 days. No intravenous lines or flow probes were available during observations. Table 1 demonstrates stable pump speed (rpm), energy consumption (Watts), and biochemistry during the 11-day pump run. Autopsy (\#9134, Leuven) demonstrated no signs of emboli or infarction in any

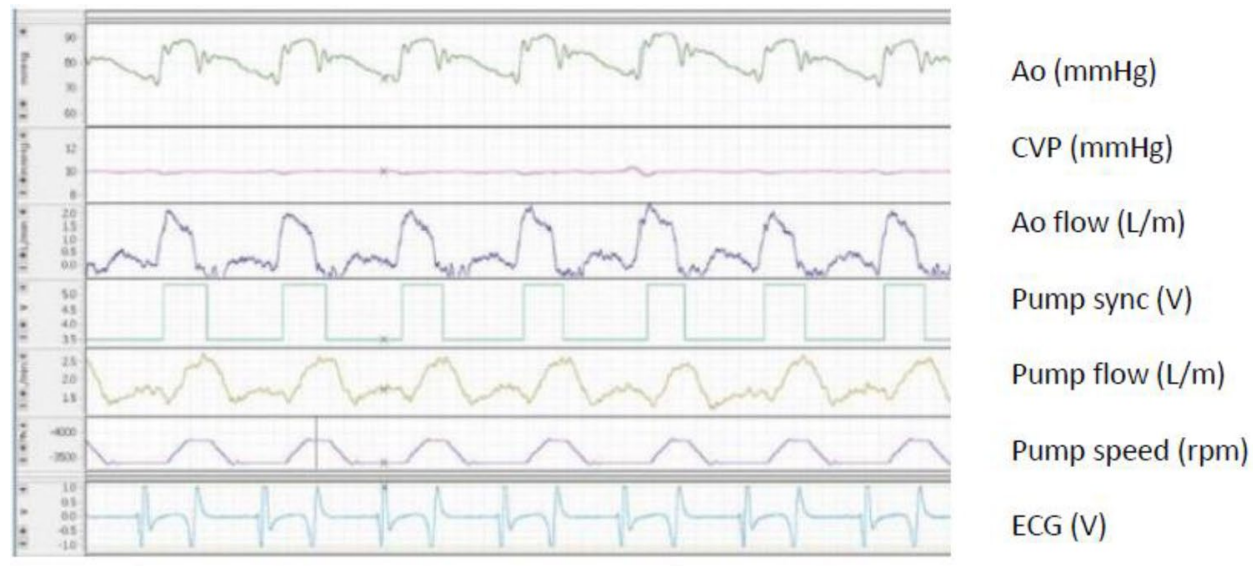

Fig. 3 Hemodynamic and circulatory variables with PulseVAD in a sedated sheep, acute study. Pulsed and synchronized mode. Ao, aortic pressure $(90 / 70 \mathrm{mmHg}$ ) measured invasively; CVP, central venous pressure $(10 \mathrm{mmHg})$ measured invasively in internal jugular vein; Ao flow, aortic flow $(0-2 \mathrm{~L} / \mathrm{m})$ measured with flow probe at proximal aorta proximal to pump outflow; Pump sync, pump synchronized to ECG; Pump flow $(1.3-2.5 \mathrm{~L} / \mathrm{m})$ measured with flow probe at pump outflow graft; Pump speed (1800-3700 rpm); ECG, electrocardio$\operatorname{gram}(\mathrm{V})$ 


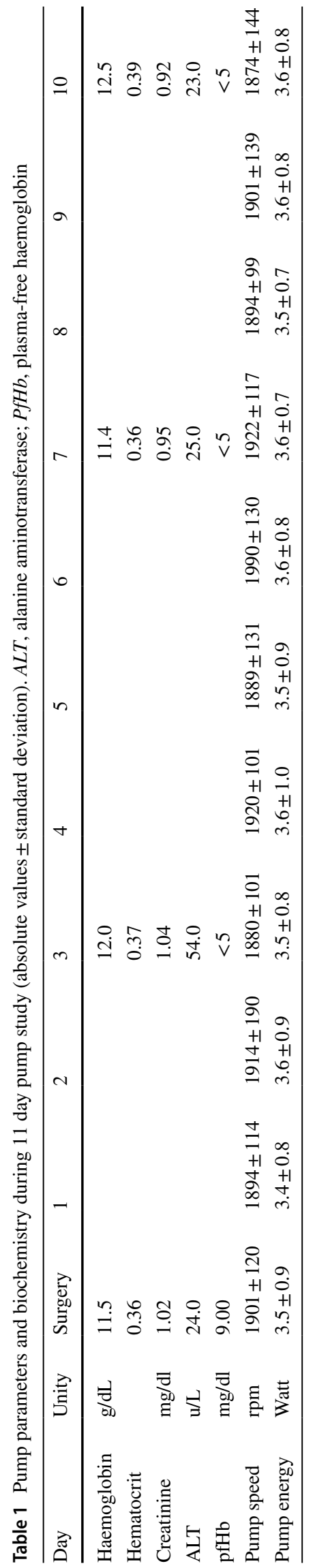

organ, and pump inspection demonstrated no deposits in the pump house. Pump-atrium interface at implantation site connection was normal, and graft aorta interface was unremarkable.

\section{Smartpump: surgical approaches for LA entry points and alternative arterial outflow sites}

For inflow cannulation of the PulseVAD in HFpEF, the LA in humans may be cannulated via a minimally invasive surgical procedure by right anterolateral thoracotomy to access the groove between the RA and LA, by left anterolateral thoracotomy to access the lateral portion of the LA between left atrial appendage and left pulmonary veins, or by upper ministernotomy to access the roof of LA, between the superior vena cava and aorta. It is important to achieve stable positioning of inflow parts without risk of suction of the LA, as well as dislocation, kinking, or compression of the graft or nearby anatomical structures. The PulseVAD has a size and configuration which allows placement in a pocket beneath the pectoral muscle, outside the ribs on either side, or alternatively in the pleural cavity. The outflow graft from the PulseVAD may be connected to the right axillary artery, ascending aorta, left axillary artery, or descending aorta at different levels as demonstrated in Fig. 1. All of these surgical approaches have advantages and disadvantages with respect to surgical technique and risks, depending on the anatomy and frailty of patients.

An anterolateral thoracotomy left side was performed in all sheep studies with direct pump inflow cannulation of left atrium between the left atrial appendage and left pulmonary veins and with the outflow graft from the pump connected to descending aorta. In- and outflow from the smartpump in such a configuration may reduce the risk of cerebral embolization compared to other alternatives mentioned above.

\section{Conclusion}

The HFpEF population is increasing in number and no therapy improving QoL and mortality is available. We have tested a new adaptive mechanical circulatory device with inflow from the LA and outflow to descending aorta. Initial animal tests have been promising, indicating that this approach may represent an effective long-term treatment for $\mathrm{HFpEF}$ patients with the potential to reduce filling pressures and increase cardiac output and thereby confer a positive effect on QoL. 
Funding Open access fundingprovided by University of Oslo (incl Oslo University Hospital).

\section{Declarations}

Competing interests Einar Gude and Arnt E Fiane are consultants for Northern Research.

Open Access This article is licensed under a Creative Commons Attribution 4.0 International License, which permits use, sharing, adaptation, distribution and reproduction in any medium or format, as long as you give appropriate credit to the original author(s) and the source, provide a link to the Creative Commons licence, and indicate if changes were made. The images or other third party material in this article are included in the article's Creative Commons licence, unless indicated otherwise in a credit line to the material. If material is not included in the article's Creative Commons licence and your intended use is not permitted by statutory regulation or exceeds the permitted use, you will need to obtain permission directly from the copyright holder. To view a copy of this licence, visit http://creativecommons.org/licenses/by/4.0/.

\section{References}

1. Heidenreich PA, Albert NM, Allen LA, Bluemke DA, Butler J, Fonarow GC et al (2013) Forecasting the impact of heart failure in the United States: a policy statement from the American Heart Association. Circ Heart Fail 6(3):606-619

2. Lam CS, Donal E, Kraigher-Krainer E, Vasan RS (2011) Epidemiology and clinical course of heart failure with preserved ejection fraction. Eur J Heart Fail 13(1):18-28

3. Oktay AA, Rich JD, Shah SJ (2013) The emerging epidemic of heart failure with preserved ejection fraction. Curr Heart Fail Rep 10(4):401-410

4. Steinberg BA, Zhao X, Heidenreich PA, Peterson ED, Bhatt DL, Cannon CP et al (2012) Trends in patients hospitalized with heart failure and preserved left ventricular ejection fraction: prevalence, therapies, and outcomes. Circulation 126(1):65-75

5. Gupta A, Bailey SR (2018) Update on devices for diastolic dysfunction: options for a no option condition? Curr Cardiol Rep 20(10):85

6. Reddy YN, Borlaug BA (2016) Heart failure with preserved ejection fraction. Curr Probl Cardiol 41(4):145-188

7. Obokata M, Reddy YNV, Pislaru SV, Melenovsky V, Borlaug BA (2017) Evidence supporting the existence of a distinct obese phenotype of heart failure with preserved ejection fraction. Circulation 136(1):6-19

8. Koh AS, Tay WT, Teng THK, Vedin O, Benson L, Dahlstrom U et al (2017) A comprehensive population-based characterization of heart failure with mid-range ejection fraction. Eur J Heart Fail 19(12):1624-1634

9. Komajda M, Lam CS (2014) Heart failure with preserved ejection fraction: a clinical dilemma. Eur Heart J 35(16):1022-1032

10. Escher A, Choi Y, Callaghan F, Thamsen B, Kertzscher U, Schweiger $M$ et al (2020) A valveless pulsatile pump for heart failure with preserved ejection fraction: hemo- and fluid dynamic feasibility. Ann Biomed Eng 48(6):1821-1836

11. Owan TE, Hodge DO, Herges RM, Jacobsen SJ, Roger VL, Redfield MM (2006) Trends in prevalence and outcome of heart failure with preserved ejection fraction. N Engl J Med 355(3):251-259

12. Plitt GD, Spring JT, Moulton MJ, Agrawal DK (2018) Mechanisms, diagnosis, and treatment of heart failure with preserved ejection fraction and diastolic dysfunction. Expert Rev Cardiovasc Ther 16(8):579-589

13. Yusuf S, Pfeffer MA, Swedberg K, Granger CB, Held P, McMurray $\mathrm{JJ}$ et al (2003) Effects of candesartan in patients with chronic heart failure and preserved left-ventricular ejection fraction: the CHARMPreserved Trial. Lancet (London, England) 362(9386):777-781

14. Gillmore JD, Damy T, Fontana M, Hutchinson M, Lachmann HJ, Martinez-Naharro A et al (2018) A new staging system for cardiac transthyretin amyloidosis. Eur Heart J 39(30):2799-2806

15. Ponikowski P, Voors AA, Anker SD, Bueno H, Cleland JG, Coats AJ et al (2016) ESC Guidelines for the diagnosis and treatment of acute and chronic heart failure: the Task Force for the diagnosis and treatment of acute and chronic heart failure of the European Society of Cardiology (ESC). Developed with the special contribution of the Heart Failure Association (HFA) of the ESC. Euro J Heart Fail 18(8):891-975

16. Lam CSP, Voors AA, de Boer RA, Solomon SD, van Veldhuisen DJ (2018) Heart failure with preserved ejection fraction: from mechanisms to therapies. Eur Heart J 39(30):2780-2792

17. Redfield MM (2016) Heart failure with preserved ejection fraction. N Engl J Med 375(19):1868-1877

18. Edelmann F, Wachter R, Schmidt AG, Kraigher-Krainer E, Colantonio C, Kamke W et al (2013) Effect of spironolactone on diastolic function and exercise capacity in patients with heart failure with preserved ejection fraction: the Aldo-DHF randomized controlled trial. JAMA 309(8):781-791

19. Zheng SL, Chan FT, Nabeebaccus AA, Shah AM, McDonagh T, Okonko DO et al (2018) Drug treatment effects on outcomes in heart failure with preserved ejection fraction: a systematic review and meta-analysis. Heart (British Cardiac Society) 104(5):407-415

20. Massie BM, Carson PE, McMurray JJ, Komajda M, McKelvie R, Zile MR et al (2008) Irbesartan in patients with heart failure and preserved ejection fraction. N Engl J Med 359(23):2456-2467

21. Cleland JG, Tendera M, Adamus J, Freemantle N, Polonski L, Taylor J (2006) The perindopril in elderly people with chronic heart failure (PEP-CHF) study. Eur Heart J 27(19):2338-2345

22. Pitt B, Pfeffer MA, Assmann SF, Boineau R, Anand IS, Claggett $B$ et al (2014) Spironolactone for heart failure with preserved ejection fraction. N Engl J Med 370(15):1383-1392

23. Solomon SD, Rizkala AR, Gong J, Wang W, Anand IS, Ge J et al (2017) Angiotensin receptor neprilysin inhibition in heart failure with preserved ejection fraction: rationale and design of the PARAGON-HF trial. JACC Heart failure 5(7):471-482

24. Solomon SD, Vaduganathan M, B LC, Packer M, Zile M, Swedberg $\mathrm{K}$ et al (2020) Sacubitril/valsartan across the spectrum of ejection fraction in heart failure. Circulation 141(5):352-61

25. Wehner GJ, Jing L, Haggerty CM, Suever JD, Leader JB, Hartzel DN et al (2020) Routinely reported ejection fraction and mortality in clinical practice: where does the nadir of risk lie? Eur Heart J 41(12):1249-1257

26. Burlacu A, Simion P, Nistor I, Covic A, Tinica G (2019) Novel percutaneous interventional therapies in heart failure with preserved ejection fraction: an integrative review. Heart Fail Rev 24(5):793-803

27. Kaye DM, Petrie MC, McKenzie S, Hasenfu $\beta$ G, Malek F, Post M et al (2019) Impact of an interatrial shunt device on survival and heart failure hospitalization in patients with preserved ejection fraction. ESC heart failure 6(1):62-69

28. Burkhoff D, Sayer G, Doshi D, Uriel N (2015) Hemodynamics of mechanical circulatory support. J Am Coll Cardiol 66(23):2663-2674

29. Combes A, Price S, Slutsky AS, Brodie D (2020) Temporary circulatory support for cardiogenic shock. Lancet (London, England) 396(10245):199-212 
30. Meyns BP, Simon A, Klotz S, Wittwer T, Schlensak C, Rega $F$ et al (2011) Clinical benefits of partial circulatory support in New York Heart Association Class IIIB and Early Class IV patients. European journal of cardio-thoracic surgery : official journal of the European Association for Cardio-thoracic Surgery 39(5):693-698

31. Fukamachi K, Horvath DJ, Karimov JH, Kado Y, Miyamoto T, Kuban BD et al (2020) Left atrial assist device to treat patients with heart failure with preserved ejection fraction: Initial in vitro study. The Journal of thoracic and cardiovascular surgery

32. Timms D, Gregory S, Hsu PL, Thomson B, Pearcy M, McNeil $\mathrm{K}$ et al (2010) Atrial versus ventricular cannulation for a rotary ventricular assist device. Artif Organs 34(9):714-720

33. Andersen MJ, Borlaug BA (2014) Invasive hemodynamic characterization of heart failure with preserved ejection fraction. Heart Fail Clin 10(3):435-444

34. Berry NC, Manyoo A, Oldham WM, Stephens TE, Goldstein RH, Waxman AB et al (2015) Protocol for exercise hemodynamic assessment: performing an invasive cardiopulmonary exercise test in clinical practice. Pulmonary circulation 5(4):610-618
35. Reddy YNV, Obokata M, Wiley B, Koepp KE, Jorgenson CC, Egbe A et al (2019) The haemodynamic basis of lung congestion during exercise in heart failure with preserved ejection fraction. Eur Heart J 40(45):3721-3730

36. Klotz S, Meyns B, Simon A, Wittwer T, Rahmanian P, Schlensak $\mathrm{C}$ et al (2010) Partial mechanical long-term support with the CircuLite Synergy pump as bridge-to-transplant in congestive heart failure. Thorac Cardiovasc Surg 58(Suppl 2):S173-S178

37. Shehab S, Macdonald PS, Keogh AM, Kotlyar E, Jabbour A, Robson D et al (2016) Long-term biventricular HeartWare ventricular assist device support-case series of right atrial and right ventricular implantation outcomes. J Heart Lung Transplant 35(4):466-473

38. Wiegmann L, Thamsen B, de Zélicourt D, Granegger M, Boës S, Schmid Daners M et al (2019) Fluid dynamics in the HeartMate 3: influence of the artificial pulse feature and residual cardiac pulsation. Artif Organs 43(4):363-376

Publisher's Note Springer Nature remains neutral with regard to jurisdictional claims in published maps and institutional affiliations. 